\title{
Influence of Full-body Water Immersion on Esophageal Motor Function and Intragastric Pressure
}

\author{
Masahito Aimi, ${ }^{1}$ Kenji Furuta, ${ }^{1 *}$ Tsukasa Saito, ${ }^{1}$ Shino Shimura, ${ }^{1}$ Kousuke Fukazawa, ${ }^{1}$ Shunji Ohara, ${ }^{1}$ Goichi Uno, ${ }^{1}$ Hiroshi \\ Tobita, ${ }^{1}$ Kyoichi Adachi ${ }^{2}$ and Yoshikazu Kinoshita ${ }^{1}$
}

Departments of ${ }^{1}$ Gastroenterology and Hepatology; and ${ }^{2}$ Clinical Nursing, Shimane University School of Medicine, Shimane, Japan

\begin{abstract}
Background/Aims
In Japan, it is customary to take a daily bath during which the body is immersed in water to the neck. During full-body immersion, hydrostatic pressure is thought to compress the chest and abdomen, which might influence esophageal motor function and intragastric pressure. However, whether water immersion has a significant influence on esophageal motor function or intragastric pressure has not been shown. The aim of this study was to clarify the influence of full-body water immersion on esophageal motor function and intragastric pressure.
\end{abstract}

\section{Methods}

Nine healthy male volunteers (mean age $40.1 \pm 2.8$ years) were enrolled in this study. Esophageal motor function and intragastric pressure were investigated using a high-resolution 36-channel manometry device.

\section{Results}

All subjects completed the study protocol. Intragastric pressure increased significantly from $4.2 \pm 1.1$ to $20.6 \pm 1.4 \mathrm{mmHg}$ with full-body water immersion, while the lower esophageal high pressure zone (LEHPZ) value also increased from $20.5 \pm 2.2$ to $40.4 \pm 3.6 \mathrm{mmHg}$, with the latter being observed regardless of dietary condition. In addition, peak esophageal peristaltic pressure was higher when immersed as compared to standing out of water.

\section{Conclusions}

Esophageal motor function and intragastric pressure were altered by full-body water immersion. Furthermore, the pressure gradient between LEHPZ and intragastric pressures was maintained at a high level, and esophageal peristaltic pressure was elevated with immersion.

(J Neurogastroenterol Motil 2012;18:194-199)

\section{Key Words}

Esophageal sphincter, lower; Gastroesophageal reflux; Peristalsis

Received: October 27, 2011 Revised: January 5, 2012 Accepted: January 10, 2012

(c) This is an Open Access article distributed under the terms of the Creative Commons Attribution Non-Commercial License (http://creativecommons. org/licenses/by-nc/3.0) which permits unrestricted non-commercial use, distribution, and reproduction in any medium, provided the original work is properly cited.

*Correspondence: Kenji Furuta, MD, PhD

Department of Gastroenterology and Hepatology, Shimane University Faculty of Medicine, 89-1 Enya-cho, Izumo-shi, Shimane 693-8501, Japan

Tel: +81-853-20-2190, Fax: +81-853-20-2187, E-mail: kfuruta@med.shimane-u.ac.jp

Financial support: This study was funded in part by Grants-in-Aid for Science Research from the Ministry of Education, Culture, Sports, Science and Technology of Japan (22590683 and 22590684).

Conflicts of interest: None. 


\section{Introduction}

It is customary for Japanese individuals to take a daily bath in the evening, during which the full body is immersed in water to the neck and the abdomen is directly exposed to hydrostatic pressure, resulting in increased intragastric pressure. On the other hand, intrathoracic pressure is kept negative by diaphragm contraction and the intercostal muscles in order to keep the lungs expanded. It is speculated that intraesophageal pressure is not augmented during immersion, thus the pressure gradient between the esophagus and stomach is thought to become elevated when the body is immersed in water. There are no known reports showing whether body immersion changes resting lower esophageal sphincter (LES) pressure. In addition, lower esophageal peristaltic contractions are also an important factor for volume clearance of refluxate, though no studies regarding changes in esophageal peristaltic contractions in the condition of whole body immersion in water have been presented. Herein, we attempted to clarify the alternations of esophageal motor functions in a state of water immersion.

High-resolution manometry, a revolutionary tool for testing esophageal functions, is useful to assess the esophageal sphincter and segmental defects in esophageal body peristalsis due to its ability to provide both panoramic and simultaneous views from the pharynx to the stomach. ${ }^{1,2}$ In the present study, we used high-resolution manometry to determine whether the lower esophageal high pressure zone (LEHPZ) rised in tandem with increased intragastric pressure and how esophageal peristaltic activity changes in water.

\section{Materials and Methods}

\section{Subjects}

Nine male healthy volunteers (mean age $40.1 \pm 2.8$ years) were enrolled in this study. None had upper gastrointestinal symptoms, history of upper gastrointestinal surgery, or were taking medications known to influence esophageal motor function. Written informed consent was obtained from each volunteer before starting the study, which was carried out in accordance with the Declaration of Helsinki. This study was approved by the Ethics Committee of Shimane University.

\section{Study Protocol}

We performed high-resolution manometric tests using a ManoScan360 ${ }^{\mathrm{TM}}$ (Sierra Scientific Instruments, California, CA, USA). The manometric catheter used with this system is $4.2 \mathrm{~mm}$ in diameter and equipped with 36 intraluminal pressure transducers at $1 \mathrm{~cm}$ intervals, which are used to continuously measure static and peristaltic pressures in the esophagus and stomach. ${ }^{3,4}$ Before performing esophageal pressure measurements, the transducers were calibrated at 0 and $100 \mathrm{mmHg}$ using externally applied pressure, according to the manufacturer's instructions. The manometric catheter was inserted in a transnasal manner using $2 \%$ lidocaine jelly (Xylocaine jelly; AstraZeneka Co., Osaka, Japan), then esophageal motor function and intragastric pressure were investigated with the subject in a fasted condition. First, resting LEHPZ and intragastric pressures were measured for 5 minutes in a standing position out of water after a 15 -minute acclimation period. For measurement of LEHPZ, the e-sleeve function of the Manoview was utilized. Next, esophageal body peristaltic contractions were measured after drinking $5 \mathrm{~mL}$ of water, which was repeated at 2-minute intervals until 5 complete contraction records were obtained. Maximal peristaltic contractions were determined and the mean of 5 separate contractions was calculated to determine the representative contraction value for each segment in each subject, as previously reported. The contractile front velocity ( $\mathrm{CFV}$ ), defined as the slope of the line connecting points on the $30 \mathrm{mmHg}$ isobaric contour at the proximal margin and the distal margin of the smooth muscle esophagus, ${ }^{5,6}$ was also determined in each subject. After determining esophageal motor activity out of water, each subject immersed their body in water to the neck in a standing position in a warm pool (approximate temperature $25^{\circ} \mathrm{C}$ ). After a 15 -minute acclimation period, intraesophageal and intragastric pressure, and $\mathrm{CFV}$ measurements were repeated in the same manner as above. Esophageal body peristaltic contractions are reported to be separated into $3 \mathrm{seg}$ ments from 1 to 3 (oral, middle and lower) by 2 contraction troughs, ${ }^{7,8}$ with the same considered to occur in both the immersed and out of water conditions, as shown in Figure 1. Identification of the upper trough is generally easy, whereas that of the lower trough is sometimes difficult. Finally, each subject drank $400-600 \mathrm{~mL}$ of a liquid nutrient $(400-600 \mathrm{kcal}$, protein 17.52-26.28 g, fat 8.92-13.38 g, sugar 62.48-93.72 g; Racol ${ }^{\mathbb{R}}$, Otsuka Pharmaceutical Factory, Tokushima, Japan), then measurement of resting pressure was repeated again out of water and with full-body immersion. 


\section{Statistical Methods}

A paired $t$ test was used for statistical comparisons between the measurements obtained in and out of water. All calculations were done with Stat-View 5.0 software (Abacus Concepts Inc., Berkeley, CA, USA) for Macintosh. Data are expressed as the
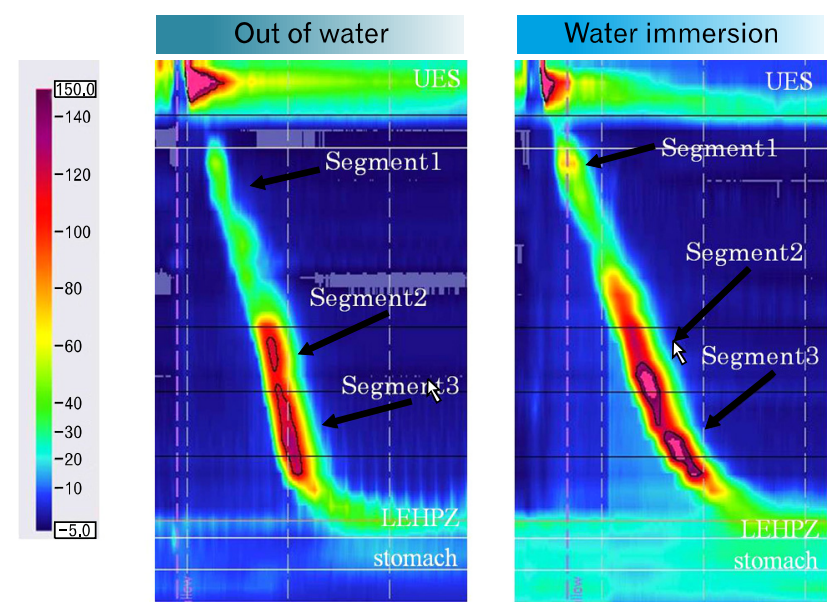

Figure 1. Esophageal body peristaltic contractions were separated into 3 segments from segments 1 to 3 (oral, middle and lower) by 2 evident troughs. In this representative subject, the separation between segments 2 and 3 out of water was observed using a $110 \mathrm{mmHg}$ isobaric contour, while that in water immersion was observed using a $130 \mathrm{mmHg}$ isobaric contour. UES, upper esophageal sphincter; LEHPZ, lower esophageal high pressure zone.

A

$\rightarrow$ Fasted codition in water

$\rightarrow-$ Fasted codition out of water

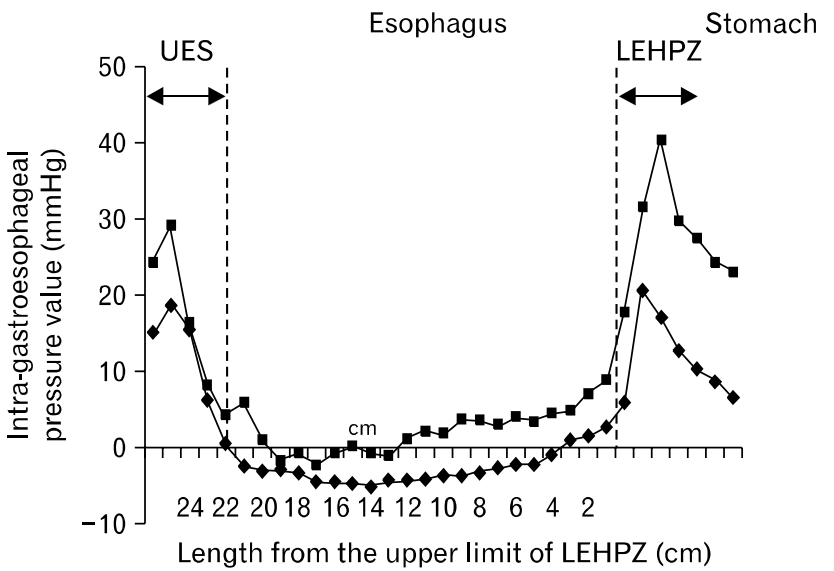

mean \pm SE. A difference level of $P<0.05$ was considered to be statistically significant.

\section{Results}

We successfully examined esophageal motor function and intragastric pressure both in air and with water immersion. The mean time of the total measuring period for each volunteer was 48.0 minutes (38.0-62.1 minutes). In a fasted state, the mean observation period time out of water was 18.2 minutes (16.0-26.0 minutes), while that in water was 15.7 minutes (13.2-21.3 minutes). In a postprandial state, the mean time out of water was 7.0 minutes (5.4-8.2 minutes), while that in water was 7.2 minutes (4.6-9.3 minutes).

\section{Resting Intra-esophageal and Intra-gastric Pres- sures After Fasting}

The mean intra-esophageal and intra-gastric resting pressures obtained while the subjects were out of and fully immersed in water are shown in Figure 1. As expected, intra-gastric pressure was elevated from $4.2 \pm 1.1 \mathrm{mmHg}$ out of water to $20.6 \pm$ $1.4 \mathrm{mmHg}$ while immersed (Fig. 2A and Table). In addition, the LEHPZ value was elevated from $20.5 \pm 2.2 \mathrm{mmHg}$ to $40.4 \pm$ $3.6 \mathrm{mmHg}$, while intra-esophageal pressures were also slightly increased. The pressure gradient between the LEHPZ and the stomach did not significantly change $(16.2 \pm 1.8 \mathrm{mmHg}$ out of

B
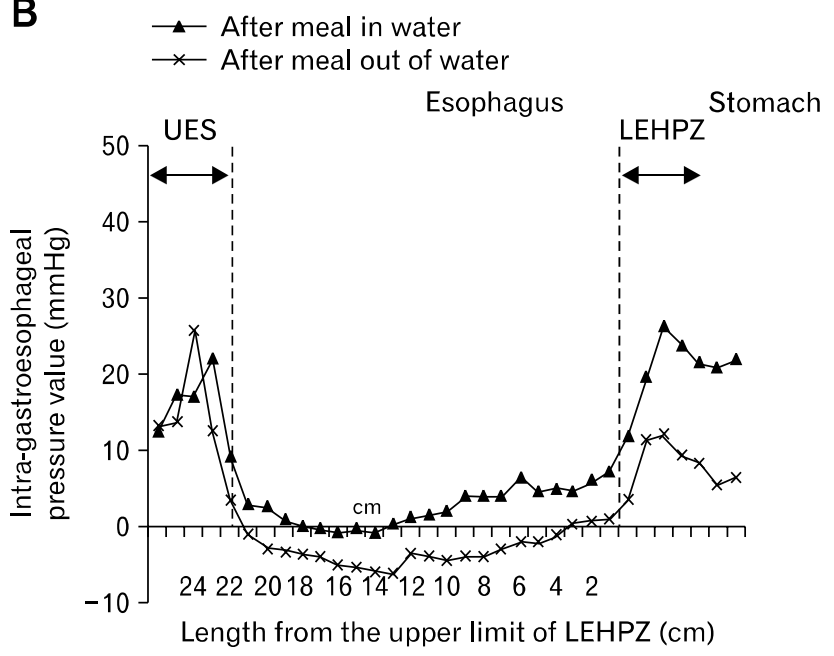

Figure 2. Intra-gastroesophageal pressure profiles in each condition. (A) Intra-gastroesophageal pressure profiles out of water $(\boldsymbol{)})$ and immersed in water $(\boldsymbol{\square})$ under the fasted condition. (B) Intra-gastroesophageal pressure profiles out of water $(\times)$ and immersed in water $(\boldsymbol{\Delta})$ during the post-prandial period. The average pressure values for all subjects for each channel are shown. Each data point represents the mean value of 9 subjects. UES, upper esophageal sphincter; LEHPZ, lower esophageal high pressure zone. 
Table. Resting Intra-gastroesophageal Pressures

\begin{tabular}{lccccc}
\hline & \multicolumn{2}{c}{ Fasting } & & \multicolumn{2}{c}{ After meal } \\
\cline { 2 - 3 } \cline { 5 - 6 } & Out of water & Water immersion & & Out of water & Water immersion \\
\hline $\mathrm{IEP}_{\text {seg1 }}(\mathrm{mmHg})$ & $-2.76 \pm 1.13$ & $-0.35 \pm 1.2^{\mathrm{a}}$ & & $-3.47 \pm 1.26$ & $0.13 \pm 1.23^{\mathrm{a}}$ \\
$\mathrm{IEP}_{\text {seg2 }}(\mathrm{mmHg})$ & $-3.89 \pm 1.36$ & $0.96 \pm 1.22^{\mathrm{a}}$ & & $-4.06 \pm 1.46$ & $0.99 \pm 1.38^{\mathrm{a}}$ \\
$\mathrm{IEP}_{\text {seg } 3}(\mathrm{mmHg})$ & $-0.76 \pm 1.43$ & $4.64 \pm 2.15^{\mathrm{a}}$ & & $0.05 \pm 1.69$ & $4.14 \pm 1.38^{\mathrm{a}}$ \\
$\mathrm{LEHPZ}(\mathrm{mmHg})$ & $20.5 \pm 2.2$ & $40.4 \pm 3.6^{\mathrm{a}}$ & & $13.3 \pm 2.0^{\mathrm{b}}$ & $27.3 \pm 2.4^{\mathrm{a}, \mathrm{b}}$ \\
$\mathrm{IGP}(\mathrm{mmHg})$ & $4.2 \pm 1.1$ & $20.6 \pm 1.4^{\mathrm{a}}$ & & $5.2 \pm 1.4$ & $17.0 \pm 2.3^{\mathrm{a}}$ \\
\hline
\end{tabular}

IEP, resting intraesophageal pressure; LEHPZ, lower esophageal high pressure zone; IGP, intragastric pressure.

${ }^{\mathrm{a}}$ Significantly different from value obtained with out of water measurement $(P<0.05)$, ${ }^{\text {b }}$ Significantly different from value obtained during fasted state $(P<0.05)$.

Data are expressed as the mean $\pm \mathrm{SE}$.

A

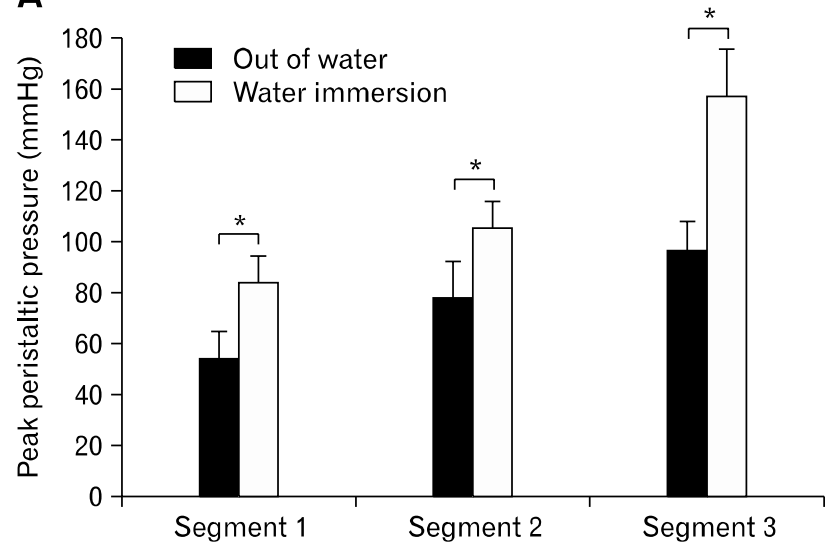

water and $19.7 \pm 2.5 \mathrm{mmHg}$ with immersion), suggesting the presence of an adequate reflux barrier at the esophago-gastric junction even with full-body immersion. There was also a small but significant increase in intra-esophageal pressure, though that rise was much smaller than that of intra-gastric pressure (Table).

\section{Esophageal Peristaltic Contractions After Fasting}

Esophageal body peristaltic contractions both out of water and during water immersion are shown in Figure 1. Because of the high intragastric pressure during water immersion, intraesophageal pressurization was observed during esophageal peristalsis with LES relaxation. Values for peristaltic pressure in each segment of the esophagus and CFV are shown in Figure 3. Peristaltic pressure during immersion was significantly higher in all segments of the esophagus, while peristalsis velocity was slower with water immersion.

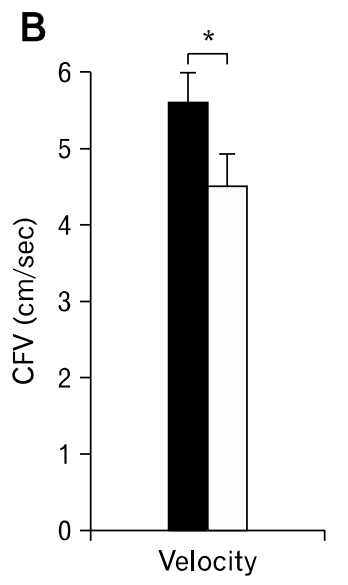

Figure 3. Peak peristaltic pressure (A) and velocity (B) in esophageal body. Data are expressed as the mean $\pm \mathrm{SE}$. Significantly different in comparison with out of water $\left({ }^{*} P<0.05\right)$. CFV, contractile front velocity.

\section{Resting Intra-esophageal and Intra-gastric Pressures After Food Intake}

After taking a liquid meal, the LEHPZ value was significantly decreased both in and out of water (Table). There were no significant differences for intraesophageal and intra-gastric resting pressures between before and after the meal (Fig. 2B), while LEHPZ was lower after consumption. The pressure gradients between the LEHPZ and stomach were not significantly different between before $(8.1 \pm 0.9 \mathrm{mmHg})$ and after $(10.3 \pm$ $2.0 \mathrm{mmHg}$ ) taking meal at water immersion condition. These results suggest the presence of post-prandial potent anti-reflux barrier that functions even when fully immersed in water.

\section{Discussion}

We investigated the influence of water immersion on esophageal motor function and intragastric pressure in healthy males using high-resolution manometry. The results demonstrated that 
esophageal motor function and intragastric pressure were influenced by full-body water immersion. In Japan, it is customary to take a daily bath in the evening with the chest and abdomen compressed by immersion to the neck, during which time the stomach is more strongly influenced by hydrostatic pressure than the intrathoracic esophagus. As expected, we found that the elevation of intragastric pressure during water immersion was higher than that of intraesophageal pressure.

In the present experiments, intragastric pressures were higher than intraesophageal pressure under all of the measurement conditions. A positive pressure gradient exists across the gastroesophageal junction and intraabdominal pressure is about 5 $\mathrm{mmHg}$ greater than intrathoracic pressure. ${ }^{9}$ We found that the resting pressure gradients between the stomach and lower esophagus out of water were 5.0 and $5.2 \mathrm{mmHg}$ in the fasting and postprandial conditions, respectively, which is in agreement with the previous report, while those during water immersion were 16.0 and $12.9 \mathrm{mmHg}$, respectively. These results suggest the possibility that gastroesophageal reflux episodes may increase because of a higher pressure gradient when immersed in water, when reflux is not prevented by the LEHPZ.

We found that the LEHPZ value was significantly increased with water immersion, though the reason cannot be deduced from our data. A possible mechanism is summarized as follows. The intrinsic smooth muscle of the distal esophagus and skeletal muscle of the crural diaphragm constitute the sphincter mechanism at the lower end of the esophagus. ${ }^{10-12}$ The crural diaphragm can externally compress the lower esophagus and augment intraesophageal pressure resulting in the prevention of reflux during inspiration when the diaphragm contracts. ${ }^{13}$ When immersed in water, the diaphragm may contract more strongly to maintain intrathoracic negative pressure in order for the lungs to be expanded, while the crural diaphragm contracts more strongly, resulting in greater external compression of the esophagus. Thus, augmented contraction of the diaphragm may explain why LEHPZ pressure rises during full-body water immersion. To test this speculation, a future dynamic study that measures not only intraesophageal gastric pressure, but also diaphragmatic crural contractions will be necessary.

Our results demonstrated for the first time that both resting LEHPZ and esophageal peristaltic pressure were elevated with full-body water immersion, though the precise mechanism is yet to be clarified. Notably, elevation of peak peristaltic pressure in the esophageal body when immersed in water is an interesting finding of our study. When wet swallowing was induced during water immersion, intraesophageal pressure below the peristaltic contraction wave was greater than that observed when out of water. When immersed, elevated intragastric pressure is directly transmitted to the esophagus during deglutitive LES relaxation, which increases resistance against peristalsis-induced food transport. To counteract such resistance, peristaltic esophageal contractions must be augmented, as shown in the present study. Therefore, the augmented esophageal body contractions observed during water immersion may be an adaptive mechanism to maintain esophageal transport for effective volume clearance of the lower esophagus.

During water immersion, intra-esophageal resting pressure rose slightly and was greater in the distal esophagus than in the proximal esophagus, as previously reported by Johnson LF et al. ${ }^{14}$ Thus, the distal esophagus may be more strongly influenced by elevated intra-gastric pressure via the transiently relaxed LES.

The present findings also indicate that the wave velocity of esophageal contractions tends to be slower with elevated peristaltic contraction pressure when immersed in water. An inverse correlation between esophageal peristaltic contractions and wave velocity has been noted in previous studies. ${ }^{7,16}$ High amplitude and slower velocity of primary peristalsis with full-body water immersion might be caused by swallow-related LES relaxation from higher intra-bolus pressure and higher intragastric pressure. Although the mechanisms are not clear, slowly elevating amplitude peristalsis may more efficiently propel esophageal contents to the stomach.

There are several limitations to our study, making it difficult to generalize the results. First, the number of volunteers was limited. In addition, since we studied only healthy volunteers, we could not determine whether the LEHPZ and esophageal peristalsis can effectively prevent reflux and clear refluxate in patients with GERD when immersed in water. Finally, the influence of hydrostatic pressure by water immersion may differ depending on water temperature, water level, and body position. A future study with a greater number of subjects including GERD patients may be necessary to clarify the effects of hydrostatic pressure, water level, water temperature and body position on esophagus motor activity during water immersion.

In conclusion, esophageal motor functions and intra-gastric pressure were altered by full-body water immersion. Furthermore, the pressure gradient between the LEHPZ and stomach was maintained at a high level, while esophageal peristaltic pressure was elevated while immersed in water. 


\section{References}

1. Herbella FA, Vicentine FP, Del Grande JC. High-resolution and conventional manometry in the assessment of the lower esophageal sphincter length. J Gastrointest Surg 2010;14:1466-1467, author reply 1468-1469.

2. Roman S, Pandolfino J, Mion F. High-resolution manometry: a new gold standard to diagnose esophageal dysmotility? Gastroenterol Clin Biol 2009;33:1061-1067.

3. Ayazi S, Hagen JA, Zehetner J, et al. The value of high-resolution manometry in the assessment of the resting characteristics of the lower esophageal sphincter. J Gastrointest Surg 2009;13:2113-2120.

4. Kahrilas PJ, Sifrim D. High-resolution manometry and impedance$\mathrm{pH} /$ manometry: valuable tools in clinical and investigational esophagology. Gastroenterology 2008;135:756-769.

5. Park MI. Recent concept in interpreting high-resolution manometry. J Neurogastroenterol Motil 2010;16:90-93.

6. Pandolfino JE, Fox MR, Bredenoord AJ, Kahrilas PJ. High-resolution manometry in clinical practice: utilizing pressure topography to classify oesophageal motility abnormalities. Neurogastroenterol Motil 2009;21:796-806.

7. Yoshida K, Furuta K, Adachi K, et al. Effects of anti-hypertensive drugs on esophageal body contraction. World J Gastroenterol 2010; 16:987-991.

8. Koshino K, Adachi K, Furuta K, et al. Effects of mosapride on esophageal functions and gastroesophageal reflux. J Gastroenterol Hepatol
2010;25:1066-1071.

9. Iwakiri K, Hayashi Y, Kotoyori M, Sugiura T, Kawakami A, Sakamoto $\mathrm{C}$. The minimum pressure of the lower esophageal sphincter, determined by the rapid pull-through method, is an index of severe reflux esophagitis. J Gastroenterol 2004;39:616-620.

10. Mittal RK, Balaban DH. The esophagogastric junction. N Engl J Med 1997;336:924-932.

11. Kinoshita Y, Furuta K, Adachi K, Amano Y. Asymmetrical circumferential distribution of esophagogastric junctional lesions: anatomical and physiological considerations. J Gastroenterol 2009;44:812818.

12. Liu J, Yamamoto Y, Schirmer BD, Ross RA, Mittal RK. Evidence for a peripheral mechanism of esophagocrural diaphragm inhibitory reflex in cats. Am J Physiol Gastrointest Liver Physiol 2000;278: G281-G288.

13. Kahrilas PJ. GERD pathogenesis, pathophysiology, and clinical manifestations. Cleve Clin J Med 2003;70:S4-S19.

14. Johnson LF, Lin YC, Hong SK. Gastroesophageal dynamics during immersion in water to the neck. J Appl Physiol 1975;38:449-454.

15. Tutuian R, Elton JP, Castell DO, Gideon RM, Castell JA, Katz PO. Effects of position on oesophageal function: studies using combined manometry and multichannel intraluminal impedance. Neurogastroenterol Motil 2003;15:63-67.

16. Iwakiri K, Sugiura T, Kotoyori M, et al. Effect of body position on lower esophageal sphincter pressure. J Gastroenterol 1999;34:305309. 